\title{
D Leech Induced Pyoderma Gangrenosum in an Ulcerative Colitis Patient: A Case Report
}

\author{
Anahita Sadeghi ${ }^{*}$, Behrouz Navabakhsh ${ }^{2}$, Niloofar Izadi Vahedi ${ }^{2}$
}

1. Digestive Disease Research Institute, Shariati hospital, Tehran University of Medical Sciences, Tehran, Iran

2. Department of Internal Medicine, Shariati hospital, Tehran University of Medical Sciences, Tehran, Iran

* Corresponding Author: Anahita Sadeghi, MD

Assistant Professor of Internal Medicine, Digestive Disease Research Institute, Tehran University of Medical Sciences, Shariati Hospital, N. Kargar Avenue., Tehran 14114-13137, Iran

Tel: +982182415104

Fax: +982182415500

Email: anahita825@gmail.com

Received: 07 Apr. 2015

Accepted: 19 Jul. 2015

\section{ABSTRACT}

Pyoderma gangrenosum (PG) is a painful skin lesion that results from excessive inflammatory response to a host of traumatic, inflammatory, or neoplastic processes in susceptible individuals. A clear pathogenetic mechanism as well as an exhaustive list of potential triggers for PG is yet to be fully characterized.

This case documents the occurrence of pyoderma gangrenosum following leech-therapy in a patient who is a known case of ulcerative colitis and it deserves attention because leeches have been part of medical armamentarium since ancient times and have re-emerged in the last century relying on their ancient charm and modern research revealing potential benefits of several bioactive substances in their saliva.

\section{KEYWORDS}

Pyoderma gangrenosum; Ulcerative colitis; Leech-therapy

Please cite this paper as:

Sadeghi A, Navabakhsh B, Izadi Vahedi N. Leech Induced Pyodermagangrenosum in a

Patient with Ulcerative Colitis: A Case Report. Middle East J Dig Dis 2015;8:63-6. DOI $: 10.15171 /$ mejdd.2016.09

\section{INTRODUCTION}

Pyoderma gangrenosum $(\mathrm{PG})$ is a rare neutrophilic dermatosis. Its incidence peaks between ages 20 and 50 and is more common in females. Nearly half of PG cases are associated with underlying diseases and conditions such as rheumatoid arthritis, inflammatory bowel disease and a broad spectrum of hematologic disorders. ${ }^{1}$ Several substances including medications, substances of abuse and cosmetics have been reported to trigger PG through either systemic use or local injection..$^{2-4}$ These reports could help in clarifying the largely unknown pathogenesis of pyoderma gangrenosum.

In contrast to the long-held ancient belief that hirudotherapy can purify the body from almost any ailment, medicinal leech is most commonly used in the treatment of venous congestion after reconstructive surgery in contemporary medicine. ${ }^{5}$ Hereby, we present a case of unjustified leech therapy for ulcerative colitis triggering several PG lesions at leech bite sites.

\section{CASE REPORT}

A 22-year-old woman with a 3-year history of ulcerative colitis was admitted to the hospital because of painful skin ulcerations on her legs (figure 1 A-C). She hadn't been taking the treatment prescribed for her ulcerative colitis and had been lost to follow-up since diagnosis. 


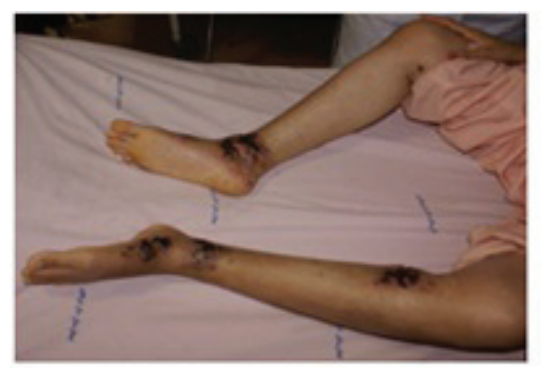

A

Fig.1: (A-C) painful skin ulcerations with purple border

B
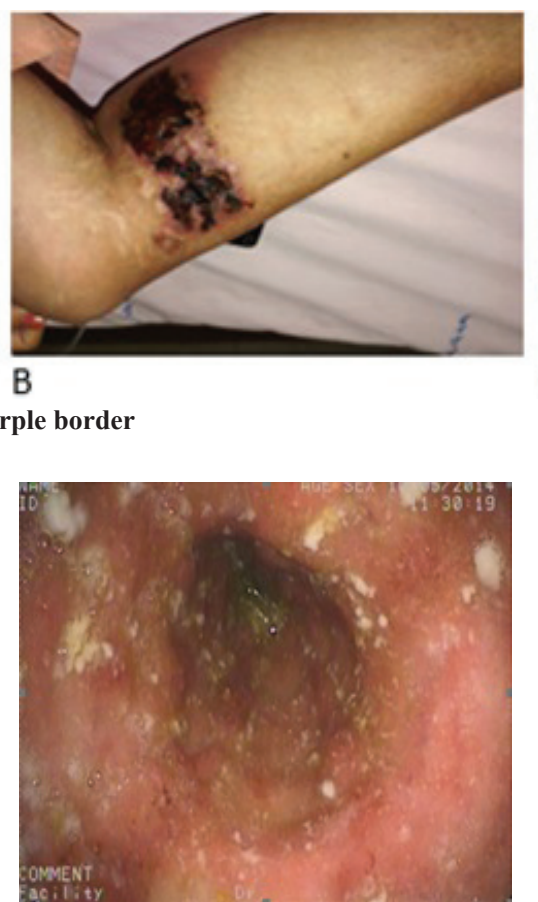

Fig.2: Diffuse ulceration with exudate and spontaneous bleeding along the rectal mucosa

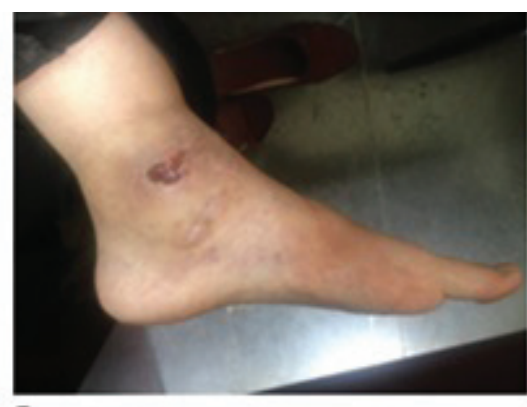

B

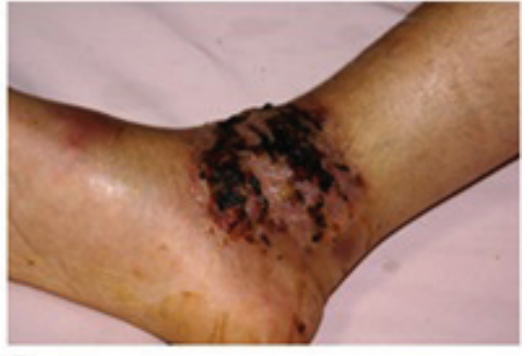

C

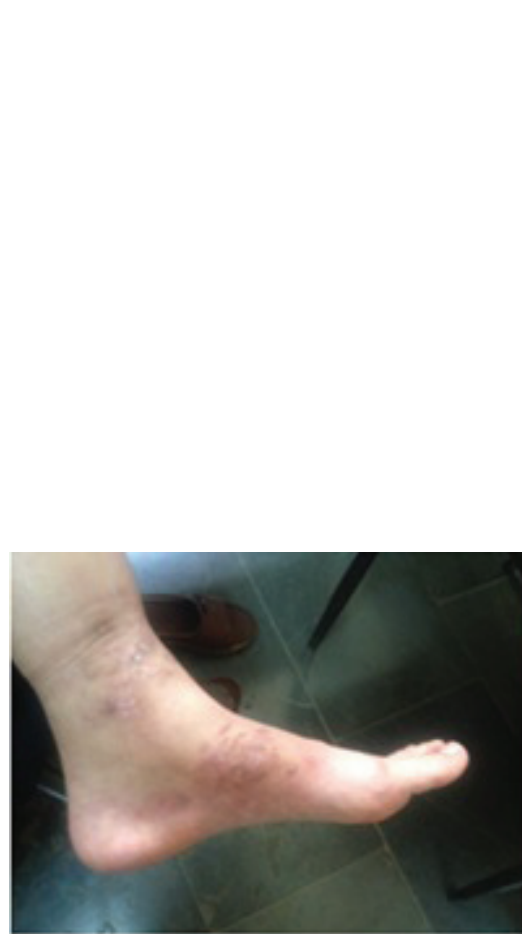

A

Fig.3: (A-C) healing of skin lesions following treatment

A few days before admission she received a single treatment of 4 to 6 locally applied leeches on her legs. After the leeches dropped from her extremities, there were some itchy areas on the leech-adhered parts of her legs. On the third day after leech therapy, she presented to the emergency department with several painful skin lesions on her legs.

Tissue cultures of the ulcerative skin lesion for bacteria, fungi and atypical mycobacteria were negative. Histopathological examination of a specimen from ulcer showed perivascular infiltrate of neutrophils and few lymphocytes in superficial and deep dermis, which confirmed the diagnosis of pyoder- ma gangrenosum. Abnormal results of laboratory tests included a hematocrit level of $16.2 \%$, CRP of $120 \mathrm{mg} / \mathrm{L}$ and erythrocyte sediment rate (ESR) of $70 \mathrm{~mm} / \mathrm{hr}$. Although she denied any history of bloody diarrhea or other symptoms of UC, colonoscopy showed diffuse ulceration with exudate and spontaneous bleeding along the rectal mucosa (figure 2). Due to active ulcerative mucosa, total colonoscopy was deferred pending proper anti-inflammatory therapy. Sulfasalazine enema ( $1 \mathrm{~g} /$ day) and prednisolone (30 mg/day) were prescribed. The skin lesions were considerably healed 6 weeks after commencement of treatment (figure $3 \mathrm{~A}-\mathrm{C}$ ). 


\section{DISCUSSION}

Pyoderma gangrenosum (PG) is a rare non-infectious neutrophil-predominant inflammation of the skin that most commonly begins as a papule, nodule, vesicle or pustule on the lower extremities and rapidly progresses to a very painful purulent ulcer with a purple undermined border. PG may also cause bullous or vegetative lesions and it may also involve peristomal, genital or extracutaneous sites. Systemic manifestations including fever, malaise, arthralgia, and myalgia may or may not accompany the skin lesions. ${ }^{1}$

In approximately $50 \%$ of patients with pyoderma gangrenosum, an underlying systemic disease is found. The most frequent systemic associations of PG are inflammatory bowel diseases, inflammatory arthropathies and hematologic disorders. The development of lesions parallels the underlying disease activity in some cases. Case reports of numerous other potential triggers and associations for PG exist: substances ranging from several medications, substances of abuse to tattoo dye and diseases ranging from rheumatologic disorders to major depression. ${ }^{1-4}$ The exact pathogenesis of PG remains to be elucidated but the frequency of its association with autoimmune disorders suggests an immunological aberrancy at the heart of its pathogenesis. The immunological theory is supported by several studies demonstrating various abnormalities in the immune cells of PG patients and helps explain certain clinical behaviors of PG including pathergy (development of PG lesions at the site of minor skin trauma), peristomal development and favorable response to immunosuppressive drugs. ${ }^{6}$

This case deserves attention because the ancient use of medicinal leech has been resurrected in the last century (including an FDA approval in 2004) with modern research revealing several potentially beneficial bioactive substances in its saliva. ${ }^{5}$ Modern medicine has found use for Leech therapy in a wide array of diseases and conditions such as CVD, reconstructive and microsurgery, malignancy, DM, infectious diseases, hearing abnormalities, arthritis and musculoskeletal pain. ${ }^{5}$

Medical literature have reported post-leeching complications such as infections, local hypersensitivity reactions including itching, blister formation, necrotic ulcers and even local tissue damage (flap death), blood loss because of prolonged hemorrhage and scars left by impaired healing of leech bites. ${ }^{5}$ However there is no report of pyoderma gangrenosum triggering by leech in the literature.

We report the first case to our knowledge where leech therapy triggered PG in a susceptible patient. This could be a case of pathergy phenomenon, which is well recognized in up to $50 \%$ of patients with $\mathrm{PG},{ }^{7}$ but since our patient did not develop any reactions at other sites of skin trauma including her IV access sites and the skin biopsy did not exacerbate her existing lesion, the mere trauma of leech bite could not sufficiently explain her skin reaction. Allergic reactions have been reported as an uncommon precipitant of pathergy phenomenon. ${ }^{8}$ A local hypersensitivity reaction to leech saliva could have induced the PG lesions in our patient.

As ancient medicine has been popularized in the recent decades in many nations, the unusual complications such as triggering pyoderma gangrenosum should be considered in mind and searched appropriately in the evaluation of our patients.

\section{ACKNOWLEDGMENT}

We thank the patient for allowing us to discuss her case in this article.

\section{CONFLICT OF INTEREST}

The authors declare no conflict of interest related to this work.

\section{REFERENCES}

1. Schadt C. Pyoderma gangrenosum: Pathogenesis, clinical features, and diagnosis. Waltham, MA: UpTo Date; 2014.

2. Roche E, Martinez-Menchon T, Sanchez-Carazo JL, Oliver $\mathrm{V}$, Alegre de Miquel V. [Two cases of eruptive pyoderma gangrenosum associated with cocaine use]. Actas Dermosifiliogr 2008;99:727-30. DOI:10.1016/S1578-2190(08)703512 .

3. Dean SM, Zirwas M. A Second Case of Sunitinib-associated. Pyoderma Gangrenosum. J Clin Aesthet Dermatol 2010;3:34-5

4. Litvinov IV, Sasseville D. Pyoderma gangrenosum 
triggered by red tattoo dye. CMAJ 2014;186:935. DOI:10.1503/cmaj.140122.

5. Abdualkader AM, Ghawi AM, Alaama M, Awang M, Merzouk A. Leech therapeutic applications. Indian J Pharm Sci 2013;75:127-37. DOI:http://dx.doi.org/10.4314/tjpr. v13i4.10.

6. Wu XR, Shen B. Diagnosis and management of parastomal pyoderma gangrenosum. Gastroenterol Rep (Oxf) 2013;1:1-8. DOI:10.1093/gastro/got013.

7. Zuo KJ, Fung E, Tredget EE, Lin AN. A systematic review of post-surgical pyoderma gangrenosum: identification of risk factors and proposed management strategy. J Plast Reconstr Aesthet Surg 2015;68:295-303.

8. Lenane P, McKenna D, Murphy GM. Pyoderma gangrenosum secondary to allergic contact dermatitis from rubber. Contact Dermatitis 1998;38:238. DOI:10.1111/j.16000536.1998.tb05734.xl. 